\title{
Crystalline Condensate in the Chiral Gross-Neveu Model
}

\author{
Gerald Dunne
}

University of Connecticut

with : Gökçe Başar, arxiv:0803.1501, Phys Rev Lett 100, 200404 (2008) arxiv:0806.xxxx 


\section{Outline}

- Gross-Neveu Model phase diagram

- chiral $\mathrm{GN}_{2}$, or $\mathrm{NJL}_{2}$

- gap equation $\rightarrow$ nonlinear Schrödinger equation

- implications for $\mathrm{NJL}_{2}$ phase diagram

- conclusions and outlook 


\section{Gross-Neveu Model}

(Gross \& Neveu, 1974)

$\mathcal{L}_{\mathrm{GN}}=\bar{\psi} i \not \partial \psi+\frac{g^{2}}{2}(\bar{\psi} \psi)^{2}$

$\mathcal{L}_{\mathrm{NJL}}=\bar{\psi} i \not \partial \psi+\frac{g^{2}}{2}\left[(\bar{\psi} \psi)^{2}+\left(\bar{\psi} i \gamma^{5} \psi\right)^{2}\right]$

$\chi \mathrm{GN}_{2}$ or $\mathrm{NJL}_{2}$

- renormalizable

- asymptotically free

- large $\mathrm{N}_{\mathrm{F}}$

- dynamical mass generation

- self-bound baryonic states

Dashen/Hasslacher/Neveu, 1975

$$
m_{B}=\frac{2}{\pi} m
$$

- $(T, \mu)$ phase diagram for $\mathrm{NJL}_{2}$ ? 


\section{$\underline{\text { lattice } \mathrm{GN}_{2} \text { model }}$}

de Forcrand/Wenger 2006

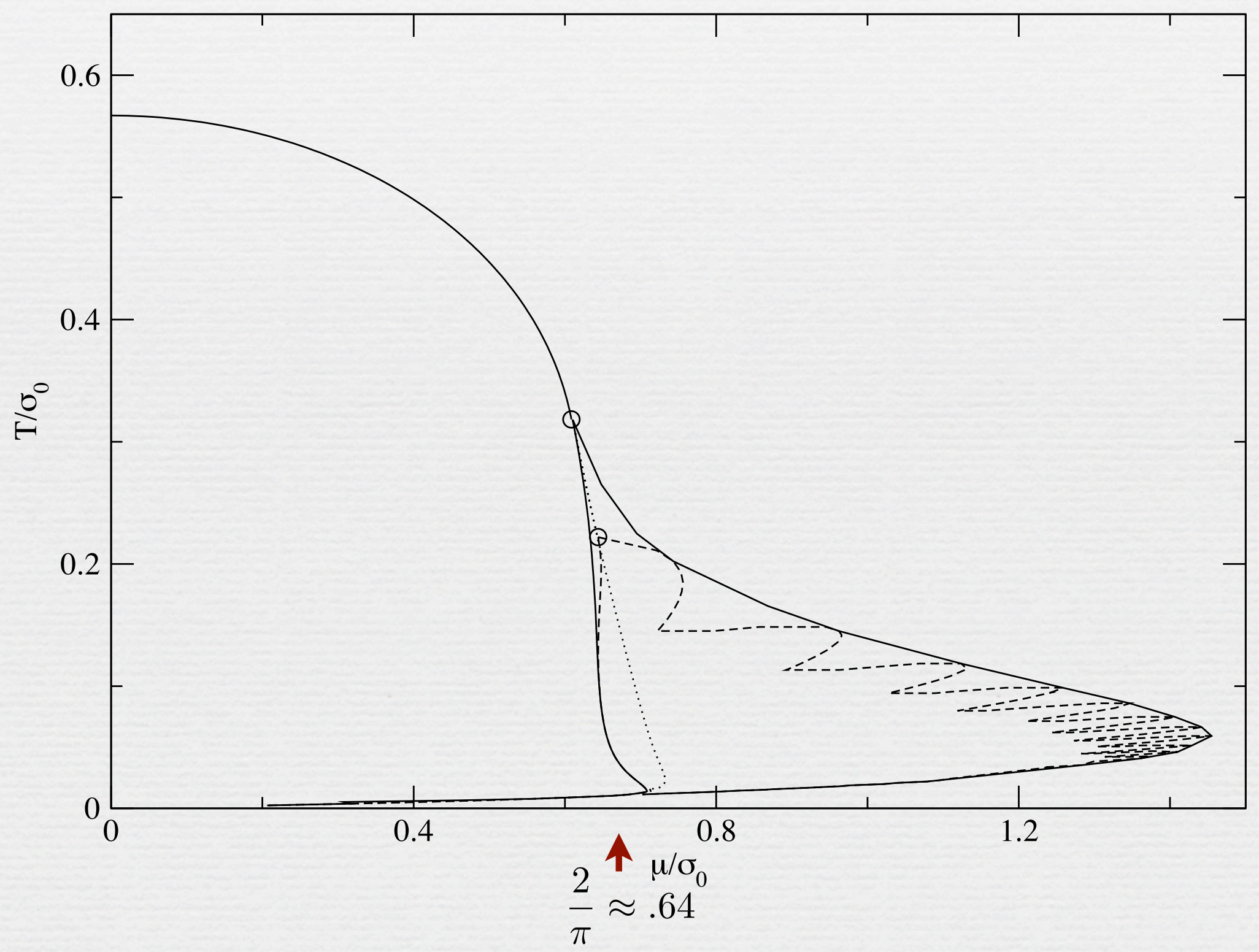




\section{Gap Equation Approach}

partition function $Z=\int \mathcal{D} \psi \exp \left\{-\int\left[\bar{\psi} \not \partial \psi-\frac{g^{2}}{2}(\bar{\psi} \psi)^{2}\right]\right\}$

effective potential

$$
V[\sigma]=\frac{1}{2 g^{2} N} \sigma^{2}-\ln \operatorname{det}[\not \partial+\sigma]
$$

gap equation

$$
\frac{\sigma(x)}{g^{2} N}=\frac{\delta}{\delta \sigma(x)} \ln \operatorname{det}[\not \partial+\sigma(x)]
$$

Dashen/Hasslacher/Neveu, 1975 : inverse scattering $\sigma^{2} \pm \sigma$ reflectionless potentials

kink state $\quad \sigma(x)=m \tanh (m x)$

Thies/Urlichs, 2005 : crystalline condensate at finite density $\sigma^{2} \pm \sigma$ finite-gap potentials

kink crystal

$$
\sigma(x)=\nu m \frac{\operatorname{sn}(m x ; \nu) \operatorname{cn}(m x ; \nu)}{\operatorname{dn}(m x ; \nu)}
$$


kink crystal $\quad \sigma(x)=\nu m \frac{\operatorname{sn}(m x ; \nu) \operatorname{cn}(m x ; \nu)}{\operatorname{dn}(m x ; \nu)}$

$$
\nu=0.999
$$

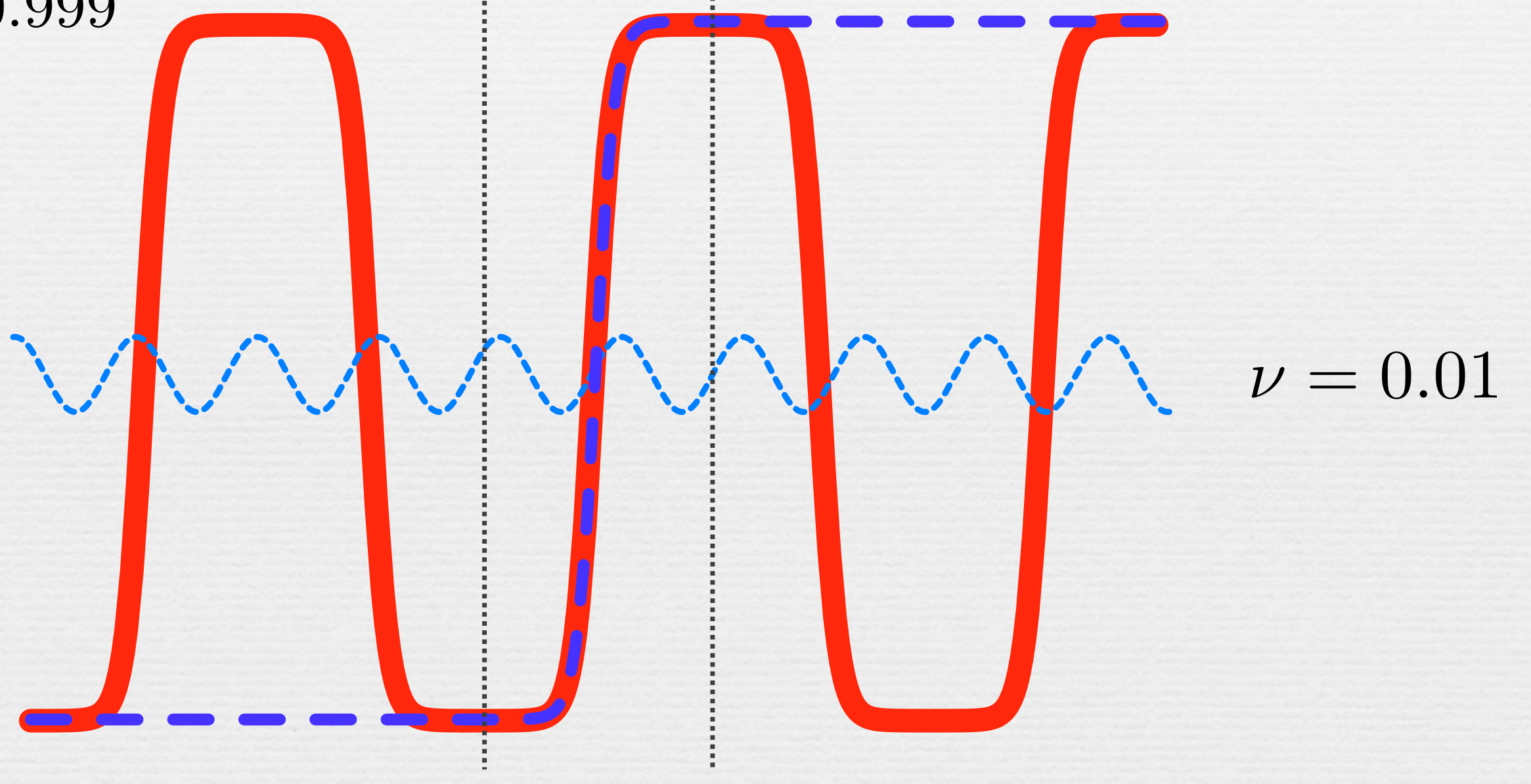




\section{Condensed matter analogues}

trans-polyacetylene $=\mathrm{GN}_{2} \quad \mathrm{Su}$, Schrieffer, Heeger, 1979

dimerization $=$ discrete chiral symmetry of GN model polaron crystal Brazovskii, 1980; Horovitz, 1981

$1 \mathrm{dim}$. Peierls-Fröhlich electron-phonon model Mertsching/Fischbeck, 1981; Belokolos et al, 1981 inhomogeneous superconductors and ferromagnetism Machida/Nakanishi, 1984 magnetic field $=\mu$

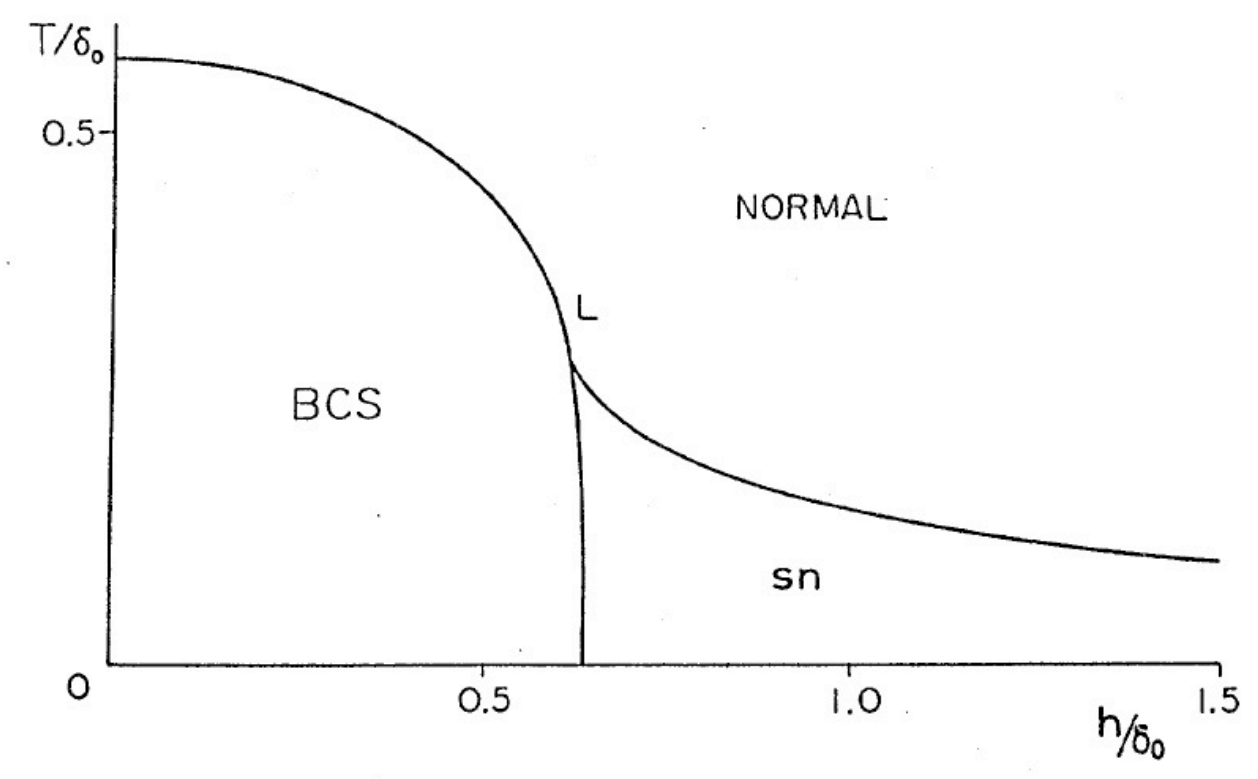




\section{$\underline{\text { Hartree-Fock approach to } \mathrm{GN}_{2} \text { gap equation }}$}

Bogoliubov/De Gennes equation : $H \psi=E \psi$

single particle states :

$$
\left(-i \gamma^{5} \frac{d}{d x}+\gamma^{0} \sigma(x)\right) \psi=E \psi
$$

self-consistent condensate :

$$
\sigma(x)=-N g^{2}\langle\bar{\psi}(x) \psi(x)\rangle
$$

$$
\sigma(x)=\nu m \frac{\operatorname{sn}(m x ; \nu) \operatorname{cn}(m x ; \nu)}{\operatorname{dn}(m x ; \nu)}
$$

soluble : "finite-gap" spectrum 


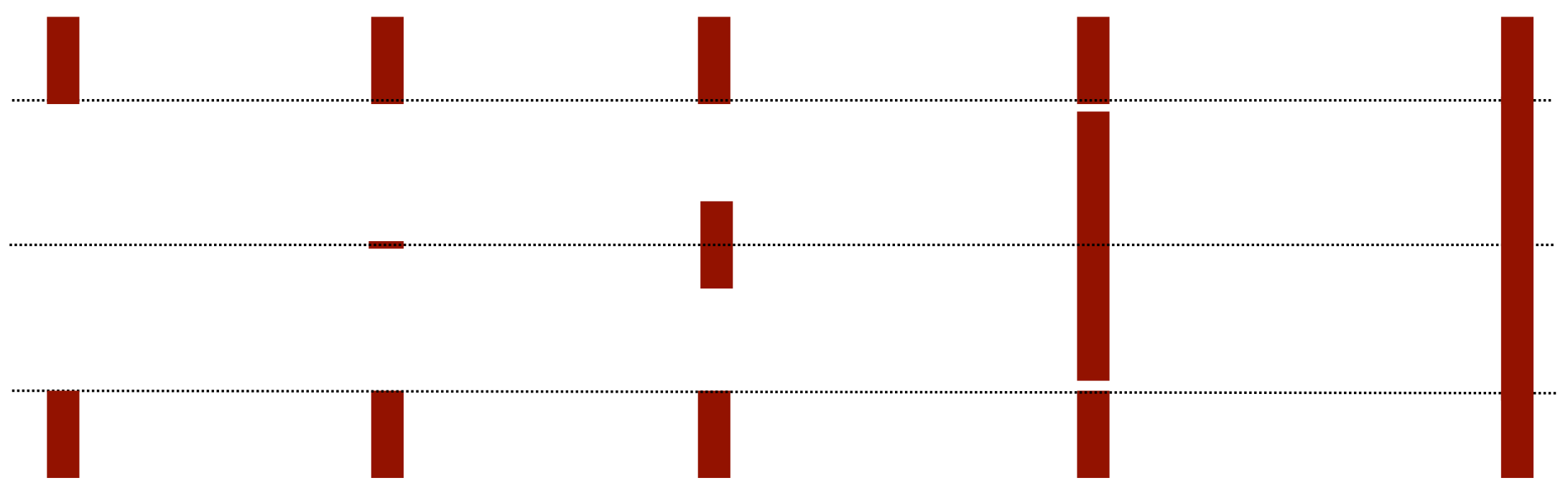

$\begin{array}{lcccc}\text { all gap } & \begin{array}{l}\text { large } \\ \text { gap }\end{array} & \text { medium } & \text { small } & \text { no gap }\end{array}$

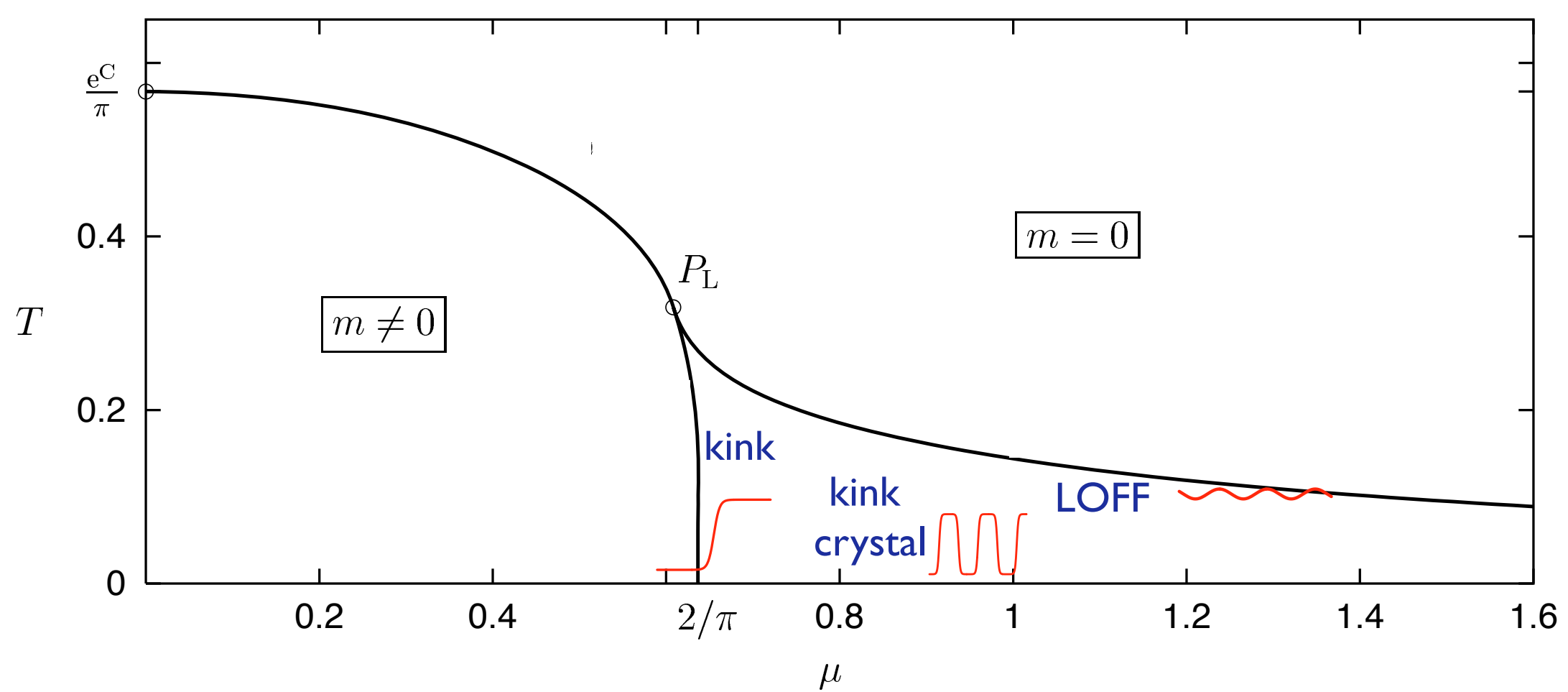




\section{chiral Gross-Neveu or $\mathrm{NJL}_{2}$}

$$
\mathcal{L}=\bar{\psi} \not \partial \psi-\frac{g^{2}}{2}\left[(\bar{\psi} \psi)^{2}+\left(\bar{\psi} i \gamma^{5} \psi\right)^{2}\right]
$$

scalar condensate $\sigma$ pseudoscalar condensate $\pi$

$$
\mathcal{L}_{\mathrm{eff}}=\bar{\psi} \not \partial \psi+\bar{\psi}\left(\sigma-i \pi \gamma^{5}\right) \psi+\frac{1}{2 g^{2}}\left(\sigma^{2}+\pi^{2}\right)
$$

gap equation(s)

$$
\begin{aligned}
& \frac{\sigma(x)}{g^{2} N}=\frac{\delta}{\delta \sigma(x)} \ln \operatorname{det}\left[\not \partial+\left(\sigma(x)-i \gamma^{5} \pi(x)\right)\right] \\
& \frac{\pi(x)}{g^{2} N}=\frac{\delta}{\delta \pi(x)} \ln \operatorname{det}\left[\not \partial+\left(\sigma(x)-i \gamma^{5} \pi(x)\right)\right]
\end{aligned}
$$

Hartree-Fock $\quad\left(-i \gamma^{5} \frac{d}{d x}+\gamma^{0} \sigma(x)+i \gamma^{1} \pi(x)\right) \psi=E \psi$

$$
\sigma(x)=-N g^{2}\langle\bar{\psi}(x) \psi(x)\rangle \quad \pi(x)=-N g^{2}\left\langle\bar{\psi}(x) i \gamma^{5} \psi(x)\right\rangle
$$




\section{phase diagram of chiral Gross-Neveu or $\mathrm{NJL}_{2}$}

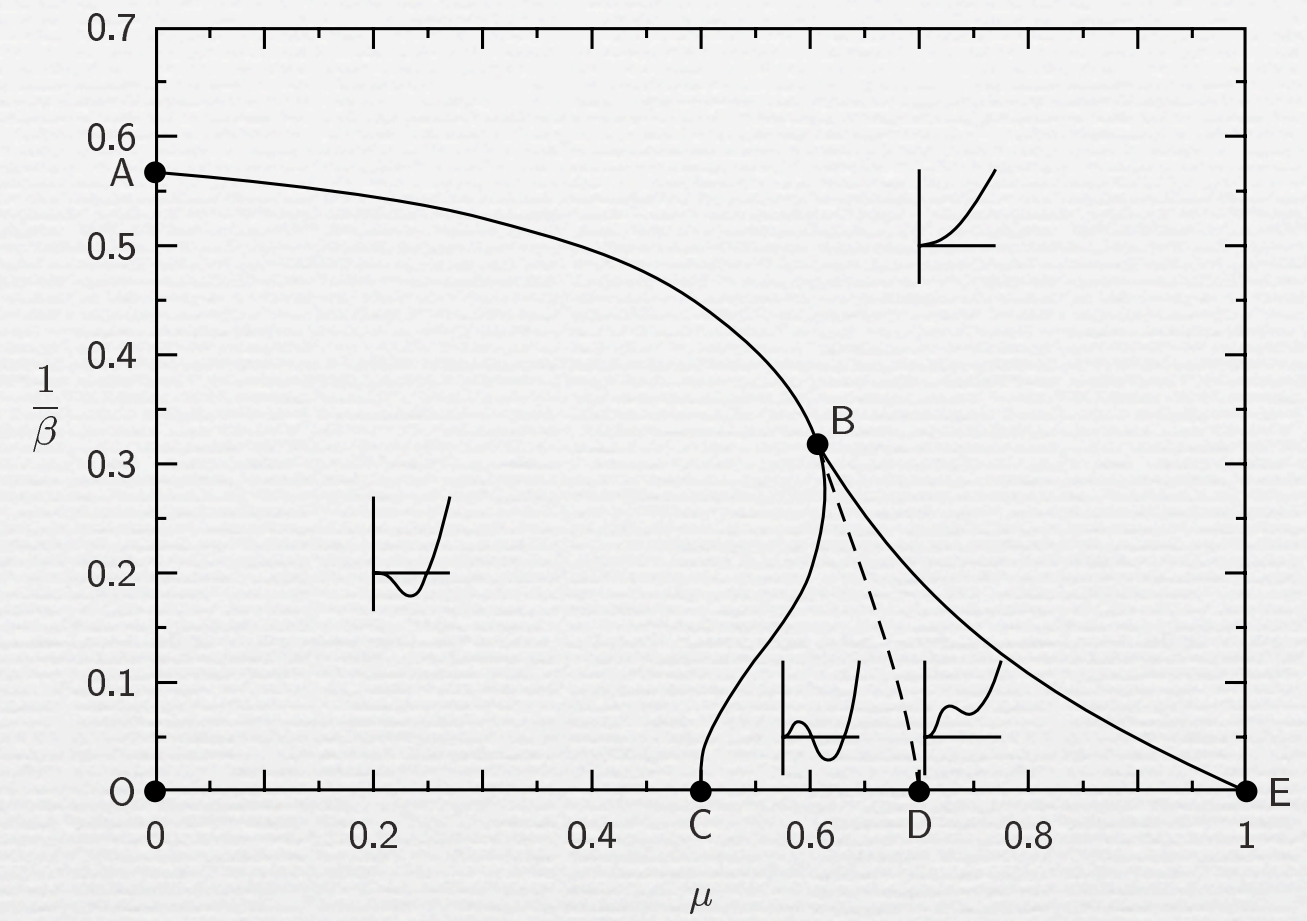

\section{Wolff, 1985}

Barducci et al, 1995

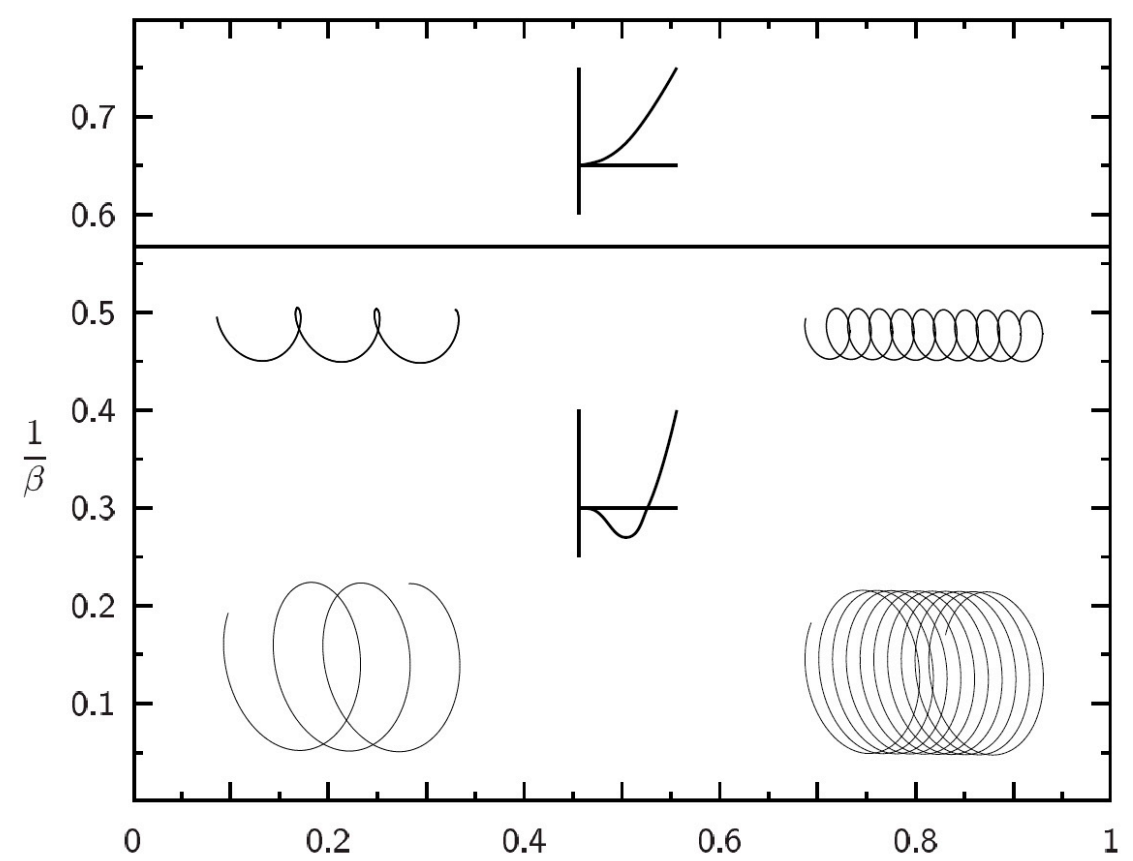

Schön/Thies, 2000

"chiral spiral"

$$
\sigma(x)-i \pi(x)=A e^{2 i \mu x}
$$


Schön/Thies, 2000

"chiral spiral"
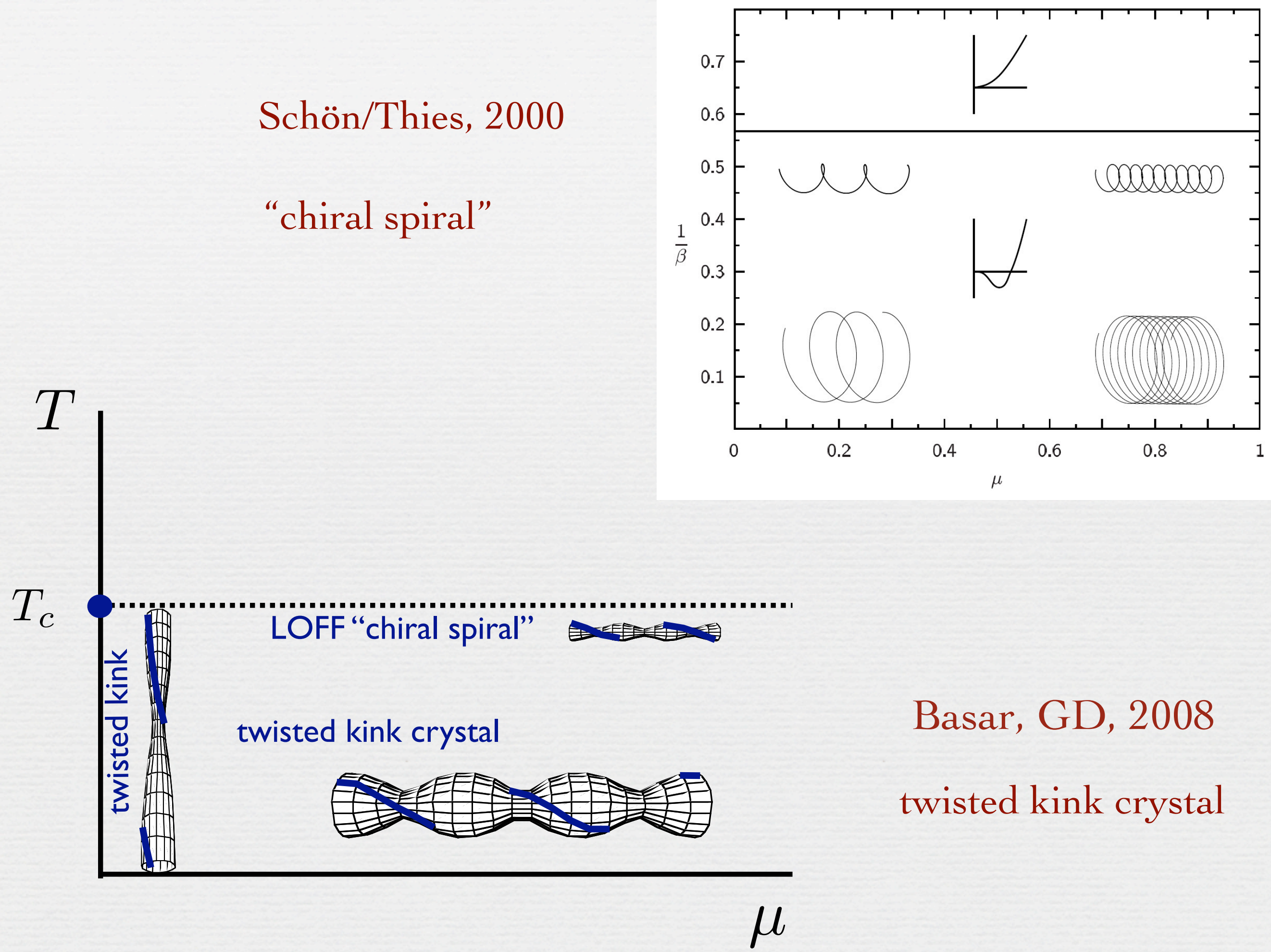

Basar, GD, 2008 twisted kink crystal 


\section{gap equation for $\mathrm{NJL}_{2}$}

Shei, 1976 : inverse scattering : reflectionless Dirac spectrum

$$
\begin{aligned}
& \sigma(x)=m\left[\cos ^{2}(\theta / 2)+\sin ^{2}(\theta / 2) \tanh (m \sin (\theta / 2) x)\right] \\
& \pi(x)=\frac{m}{2} \sin (\theta)[1-\tanh (m \sin (\theta / 2) x)] \\
& \text { twisted kink state } \quad 0 \leq \theta \leq 2 \pi
\end{aligned}
$$

Basar \& GD, 2008 : twisted kink crystal finite-gap Dirac spectrum

twisted kink crystal

$\sigma(x)-i \pi(x)=-A \frac{\sigma\left(A x+i \mathbf{K}^{\prime}-i \theta / 2\right)}{\sigma\left(A x+i \mathbf{K}^{\prime}\right) \sigma(i \theta / 2)} e^{\left[i A x(-i \zeta(i \theta / 2)+i \mathrm{~ns}(i \theta / 2))+i \theta \eta_{3} / 2\right]}$

$$
0 \leq \theta \leq 4 \mathbf{K}^{\prime}
$$




\section{twisted kink crystal}

$$
\Delta(x)=\sigma(x)-i \pi(x) \quad \Delta(x)=M(x) e^{i \chi(x)}
$$

complex kink

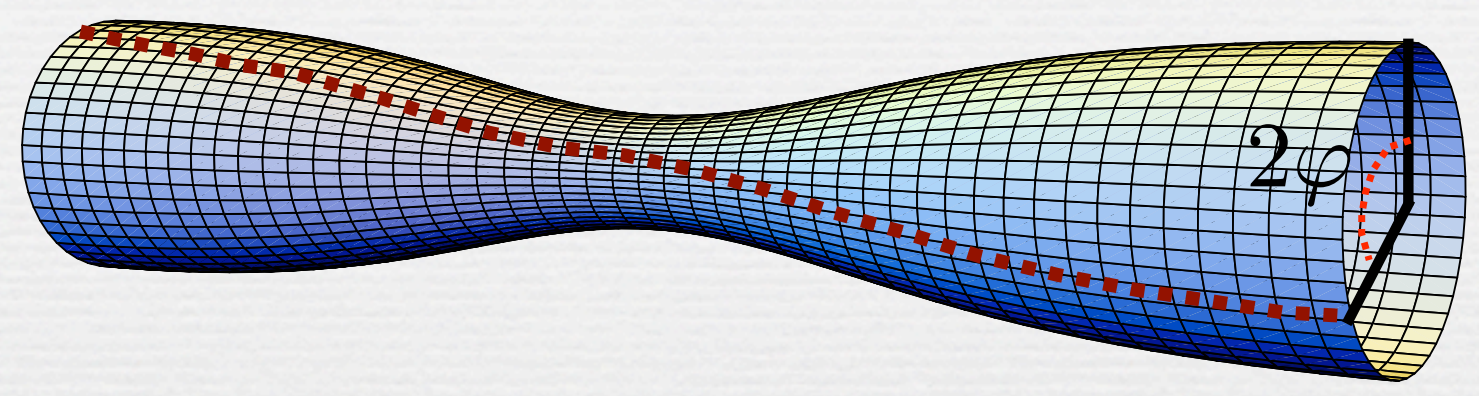

real kink

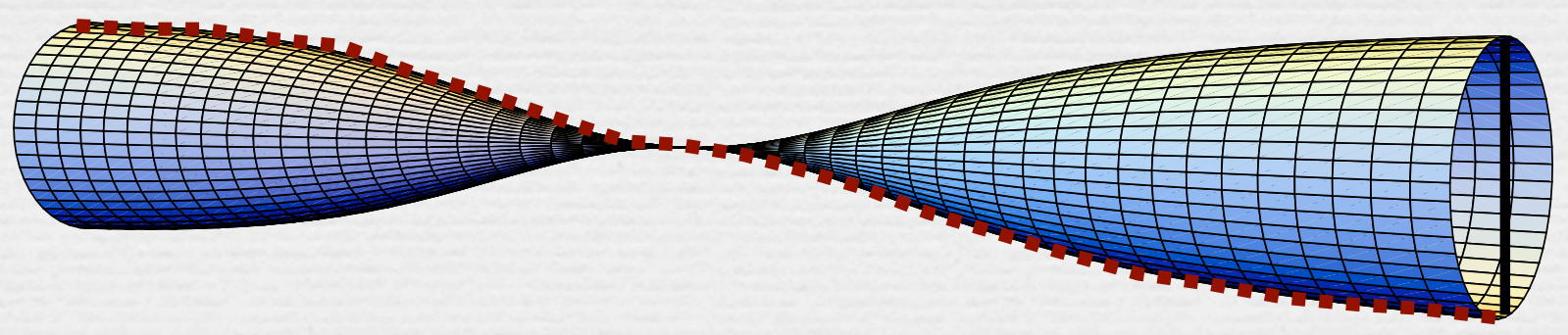

$2 \varphi=\pi$

"chiral spiral" 


\section{twisted kink crystal}

$$
\Delta(x)=\sigma(x)-i \pi(x) \quad \Delta(x)=M(x) e^{i \chi(x)}
$$

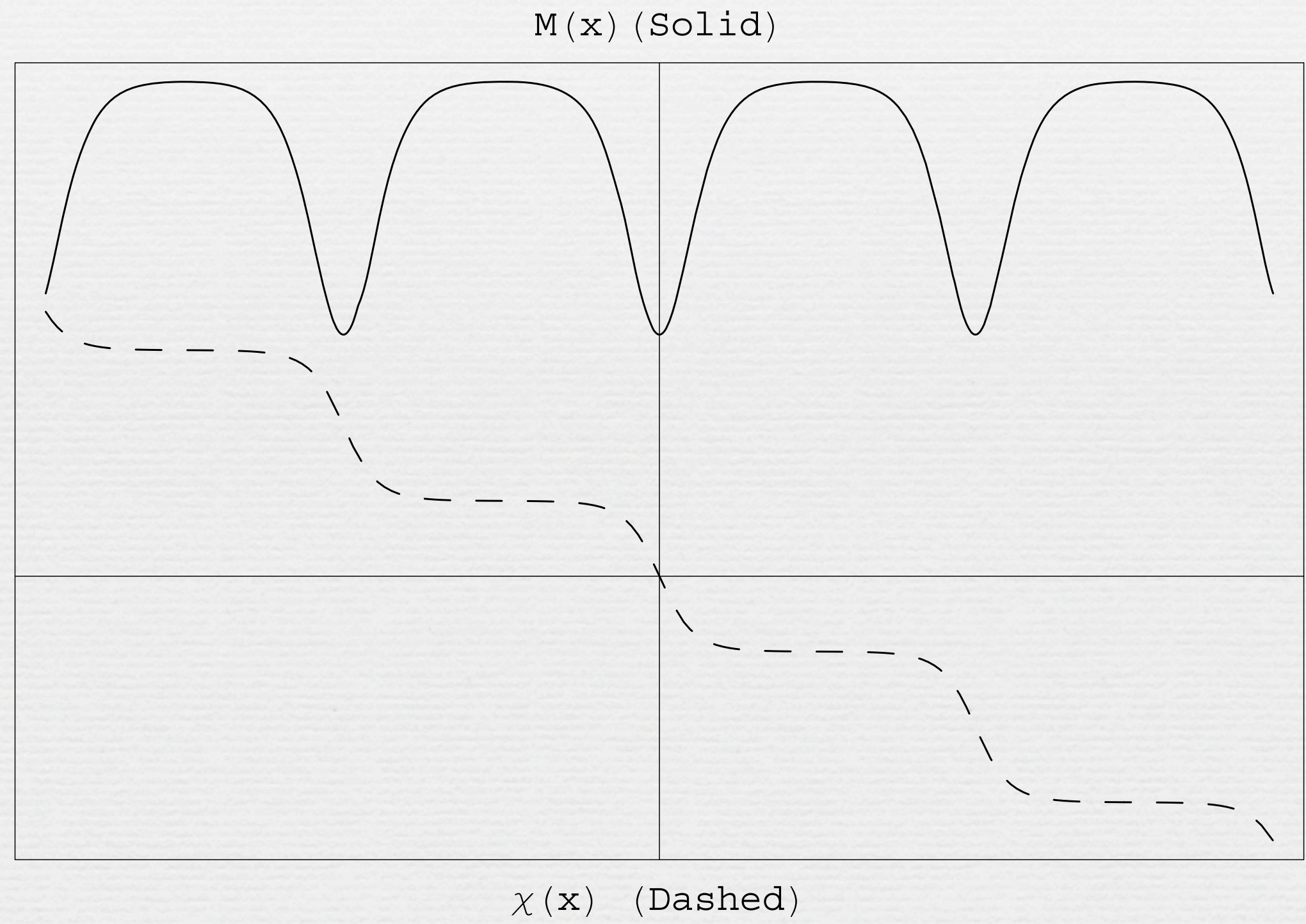


complex kink crystal
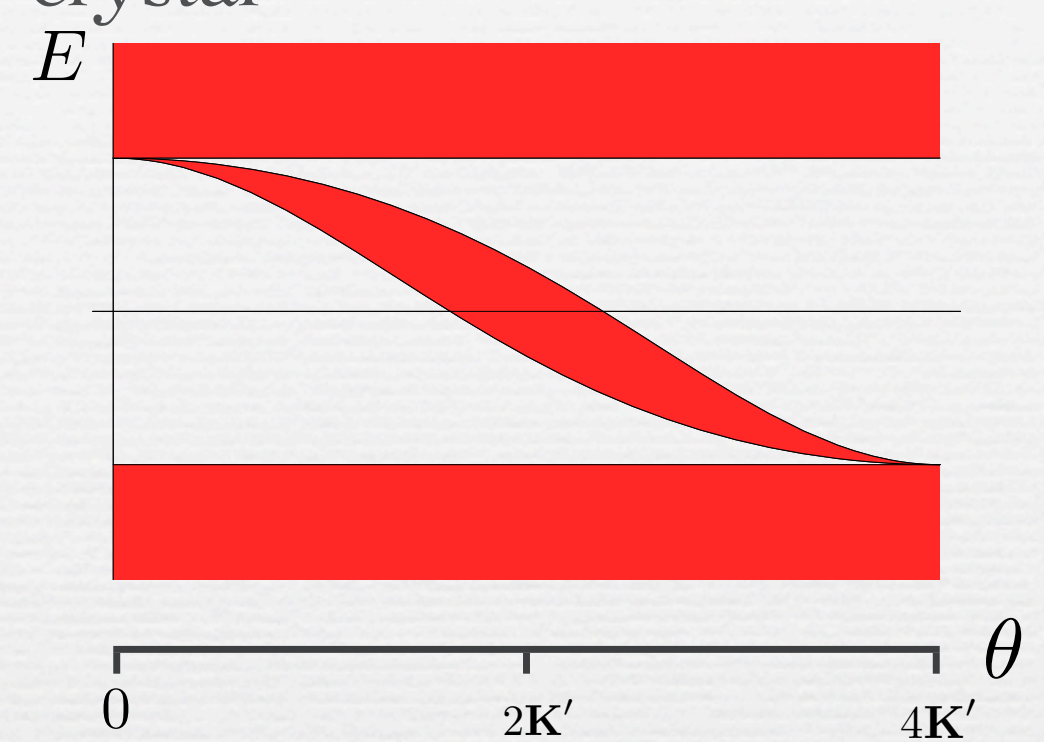

$\theta=2 \mathbf{K}^{\prime}$

real kink

crystal

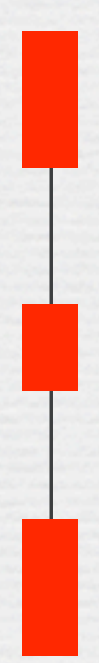

single-particle spectrum

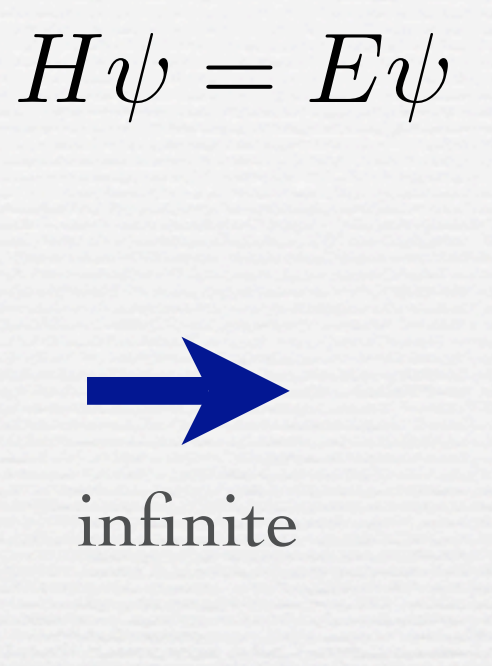

complex kink
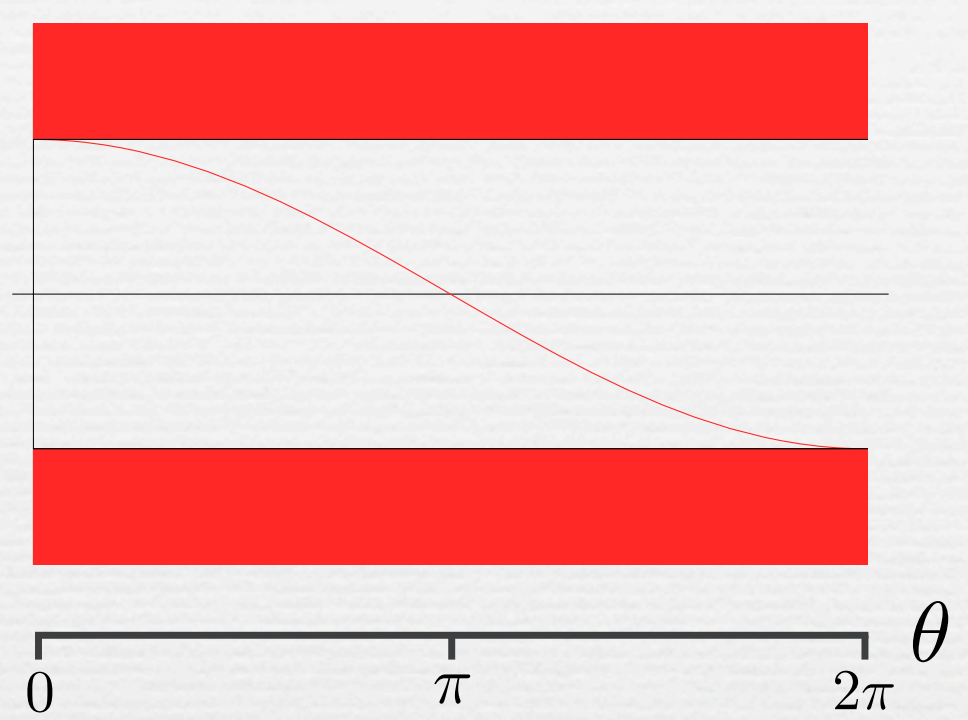

$y \theta=\pi$

real kink

infinite

period

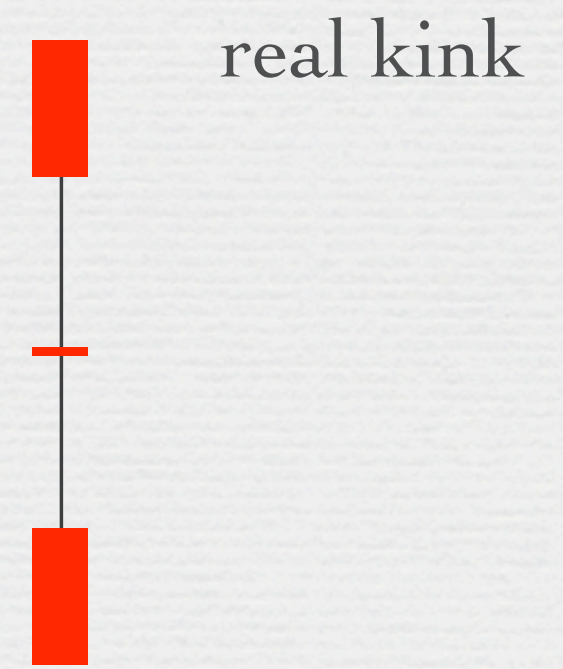




\section{solving the (complex) gap equation}

$$
\frac{\Delta(x)}{g^{2} N}=\frac{\delta}{\delta \Delta^{*}(x)} \ln \operatorname{det}\left[\not \partial+\left(\sigma(x)-i \gamma^{5} \pi(x)\right)\right]
$$

$H=-i \gamma^{5} \frac{d}{d x}+\gamma^{0} \sigma(x)+i \gamma^{1} \pi(x)=\left(\begin{array}{cc}-i \frac{d}{d x} & \Delta(x) \\ \Delta^{*}(x) & i \frac{d}{d x}\end{array}\right)$

\section{Bogoliubov/de Gennes hamiltonian}

resolvent : Gorkov Green's function

$$
R(x ; E) \equiv\left\langle x\left|\frac{1}{H-E}\right| x\right\rangle
$$

spectral function $\quad \rho(E)=\frac{1}{\pi} \operatorname{Im} \int d x \operatorname{tr} R(x ; E+i \epsilon)$ 
$\underline{\text { resolvent }}$

$$
\begin{array}{cc}
R=R^{\dagger} & \operatorname{tr}\left(R \sigma_{3}\right)=0 \\
R^{\prime} \sigma_{3}=i\left[\left(\begin{array}{cc}
E & -\Delta \\
\Delta^{*} & -E
\end{array}\right), R \sigma_{3}\right]
\end{array}
$$

$R(x ; E) \equiv\left\langle x\left|\frac{1}{H-E}\right| x\right\rangle$

$\operatorname{det} R=\frac{1}{4}$

Dik'ii equation

Eilenberger equation

two views of gap equation

$$
\begin{aligned}
& \text { ln det }\left[\not \partial+\left(\sigma(x)-i \gamma^{5} \pi(x)\right)\right]=\int d E \rho(E) \frac{1}{\beta} \ln \left(1+e^{-\beta(E-\mu)}\right) \\
& \Delta(x)=-N g^{2} \int d E \operatorname{tr}\left[\left(\gamma^{0}\left(\mathbf{1}+\gamma^{5}\right)\right) R(x ; E)\right] \\
& R(x ; E)=\mathcal{N}(E)\left(\begin{array}{cc}
a(E)+|\Delta(x)|^{2} & b(E) \Delta(x)-i \Delta^{\prime}(x) \\
b(E) \Delta^{*}(x)+i \Delta^{\prime *}(x) & a(E)+|\Delta(x)|^{2}
\end{array}\right) \\
& \Delta^{\prime \prime}-2|\Delta|^{2} \Delta+i(b-2 E) \Delta^{\prime}-2(a-E b) \Delta=0 \\
& \text { nonlinear Schrödinger equation (NLSE) }
\end{aligned}
$$


gap equation

\section{NLSE}

$$
\frac{\Delta(x)}{g^{2} N}=\frac{\delta}{\delta \Delta^{*}(x)} \ln \operatorname{det}\left[\not \partial+\left(\sigma(x)-i \gamma^{5} \pi(x)\right)\right]
$$

ansatz, from gap equation

$$
\begin{array}{r}
R(x ; E)=\mathcal{N}(E)\left(\begin{array}{cc}
a(E)+|\Delta(x)|^{2} & b(E) \Delta(x)-i \Delta^{\prime}(x) \\
b(E) \Delta^{*}(x)+i \Delta^{\prime *}(x) & a(E)+|\Delta(x)|^{2}
\end{array}\right) \\
\downarrow \text { Eilenberger equation } \\
\Delta^{\prime \prime}-2|\Delta|^{2} \Delta+i(b-2 E) \Delta^{\prime}-2(a-E b) \Delta=0
\end{array}
$$

NLSE : exactly soluble 


\section{NLSE : examples}

$$
\Delta^{\prime \prime}-2|\Delta|^{2} \Delta+i(b-2 E) \Delta^{\prime}-2(a-E b) \Delta=0
$$

DHN kink $\Delta=\tanh (x)$

Thies's kink crystal

$$
\Delta^{\prime \prime}-2 \Delta^{3}+2 \Delta=0
$$

$$
\begin{aligned}
& \Delta(x)=\nu \frac{\operatorname{sn}(x ; \nu) \operatorname{cn}(x ; \nu)}{\operatorname{dn}(x ; \nu)} \\
& \Delta^{\prime \prime}-2 \Delta^{3}-2(\nu-2) \Delta=0
\end{aligned}
$$

Shei's twisted kink $\quad \Delta(x)=\frac{\cosh (\sin (\theta / 2) x-i \theta / 2)}{\cosh (\sin (\theta / 2) x)} e^{i \theta / 2}$

$$
\Delta^{\prime \prime}-2|\Delta|^{2} \Delta-2 i \cos (\theta / 2) \Delta^{\prime}+2 \Delta=0
$$

chiral spiral $\Delta=e^{2 i \mu x} \quad \Delta^{\prime \prime}-2|\Delta|^{2} \Delta-4 i \mu \Delta^{\prime}-2\left(2 \mu^{2}-1\right) \Delta=0$

twisted kink crystal $\Delta=A \frac{\sigma\left(A x+i \mathbf{K}^{\prime}-i \theta / 2\right)}{\sigma\left(A x+i \mathbf{K}^{\prime}\right)} e^{i \lambda x}$

$\Delta^{\prime \prime}-2|\Delta|^{2} \Delta-i(2 A i \mathrm{~ns}(i \theta / 2)) \Delta^{\prime}-A^{2}\left(3 \mathcal{P}(i \theta / 2)-\mathrm{ns}^{2}(i \theta / 2)\right) \Delta=0$ 


\section{results from resolvent/NLSE approach}

exact solution of gap equation

exact resolvent and spectral function

contains all previous cases, and new complex crystal new exact crystalline solution to Eilenberger equation new exact solution to Bogoliubov/de Gennes equation 


\section{Density of States}

BdG equation : $\quad H \psi=E \psi \quad H(x)=\left(\begin{array}{cc}-i \frac{d}{d x} & \Delta(x) \\ \Delta^{*}(x) & i \frac{d}{d x}\end{array}\right)$

quasi-periodicity : $\quad \Delta(x+L)=e^{2 i \varphi} \Delta(x)$

$$
H(x+L)=e^{i \varphi \gamma^{5}} H(x) e^{-i \varphi \gamma^{5}}
$$

exact single particle "Bloch" states :

$$
\psi_{ \pm}(x+L)=e^{ \pm i k L} e^{i \varphi \gamma^{5}} \psi_{ \pm}(x)
$$

density of states:

$$
\frac{d k}{d E}=\frac{1}{L} \int_{\text {period }} \operatorname{tr} R(x ; E)
$$




\section{$\underline{\text { Implications for phase diagram of } \mathrm{NJL}_{2}}$}

exact spectral function : exact free energy

(approximate) Ginzburg-Landau approach:

$$
\begin{aligned}
\mathcal{L}_{G L}= & c_{0}+c_{2}|\Delta|^{2}+c_{3} \operatorname{Im}\left[\Delta\left(\Delta^{\prime}\right)^{*}\right]+c_{4}\left[|\Delta|^{4}+\left|\Delta^{\prime}\right|^{2}\right] \\
& +c_{5} \operatorname{Im}\left[\left(\Delta^{\prime \prime}-3|\Delta|^{2} \Delta\right)\left(\Delta^{\prime}\right)^{*}\right] \\
+ & c_{6}\left[2|\Delta|^{6}+8|\Delta|^{2}\left|\Delta^{\prime}\right|^{2}+2 \operatorname{Re}\left(\left(\Delta^{\prime}\right)^{2}\left(\Delta^{*}\right)^{2}\right)+\left|\Delta^{\prime \prime}\right|^{2}\right]
\end{aligned}
$$

real $(\mathrm{GN})$ case : tricritical point: $\mathrm{c}_{2}=\mathrm{C}_{4}=0$

complex (NJL) case : tricritical point: $\mathrm{c}_{2}=\mathrm{c}_{3}=0$

$$
\Delta^{\prime \prime}-2|\Delta|^{2} \Delta-i \frac{c_{3}}{c_{4}} \Delta^{\prime}-\frac{c_{2}}{c_{4}} \Delta=0 \quad \text { NLSE } !
$$




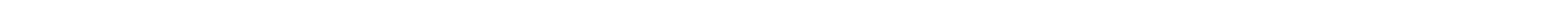




\title{
gap equation to all-orders in Ginzburg-Landau
}

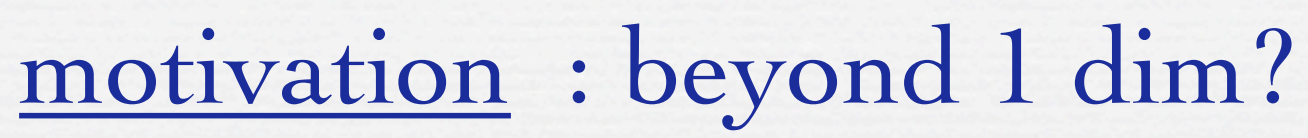

$$
\mathcal{L}_{\mathrm{GL}}=\sum_{n} c_{n}(T, \mu) a_{n}(x)
$$

$\mathcal{L}_{\mathrm{GL}}=c_{0}+c_{2}|\Delta|^{2}+c_{3} \operatorname{Im}\left[\Delta\left(\Delta^{\prime}\right)^{*}\right]+c_{4}\left[|\Delta|^{4}+\left|\Delta^{\prime}\right|^{2}\right]$

$+c_{5} \operatorname{Im}\left[\left(\Delta^{\prime \prime}-3|\Delta|^{2} \Delta\right)\left(\Delta^{\prime}\right)^{*}\right]$

$+c_{6}\left[2|\Delta|^{6}+8|\Delta|^{2}\left|\Delta^{\prime}\right|^{2}+2 \operatorname{Re}\left(\left(\Delta^{\prime}\right)^{2}\left(\Delta^{*}\right)^{2}\right)+\left|\Delta^{\prime \prime}\right|^{2}\right]+\ldots$

NLSE $\rightarrow$ entire hierarchy satisfied

$$
\left[a_{n}(x)\right]_{N L S E}=\alpha_{n}|\Delta(x)|^{2}+\beta_{n}
$$




\section{Conclusions}

- complete solution of gap equation for chiral $\mathrm{GN}_{2} / \mathrm{NJL}_{2}$

- gap equation reduced to NLSE

- Ginzburg-Landau : chiral crystalline phase

- full, exact, thermodynamics \& phase diagram ?

- lattice $\mathrm{NJL}_{2}$ at finite density and temperature?

- massive $\mathrm{NJL}_{2}$ ?

- higher dimensions? 


\section{verifying the gap equation}

two views of gap equation

$\ln \operatorname{det}\left[\not \partial+\left(\sigma(x)-i \gamma^{5} \pi(x)\right)\right]=\int d E \rho(E) \frac{1}{\beta} \ln \left(1+e^{-\beta(E-\mu)}\right)$

$\Delta(x)=-N g^{2} \int d E \operatorname{tr}\left[\left(\gamma^{0}\left(\mathbf{1}+\gamma^{5}\right)\right) R(x ; E)\right]$

$R(x ; E)=\mathcal{N}(E)\left(\begin{array}{cc}a(E)+|\Delta(x)|^{2} & b(E) \Delta(x)-i \Delta^{\prime}(x) \\ b(E) \Delta^{*}(x)+i \Delta^{\prime *}(x) & a(E)+|\Delta(x)|^{2}\end{array}\right)$

$\int \mathcal{N}(E) d E=0 \quad \rightarrow \frac{\theta}{4 \mathbf{K}^{\prime}}=$ filling fraction

cf. Shei, $1976 \frac{\theta}{2 \pi}=$ filling fraction

$N g^{2} \int \mathcal{N}(E) E d E=1$

vacuum gap equation 


\section{properties of resolvent $\quad R(x ; E) \equiv\left\langle x\left|\frac{1}{H-E}\right| x\right\rangle$} resolvent in terms of independent spinor solutions

$$
R=\frac{1}{2 W}\left(\psi_{1} \psi_{2}^{T}+\psi_{2} \psi_{1}^{T}\right) \sigma_{1}
$$

Wronskian $\quad W \equiv-i \psi_{1}^{T} \sigma_{2} \psi_{2}$

$$
\begin{gathered}
R=R^{\dagger} \quad \operatorname{tr}\left(R \sigma_{3}\right)=0 \quad \operatorname{det} R=\frac{1}{4} \\
R=\frac{1}{2}\left(\begin{array}{cc}
a & b \\
b^{*} & a
\end{array}\right) \quad a^{2}-|b|^{2}=1 \\
\psi^{\prime}=i \sigma_{3}\left(\begin{array}{cc}
E & -\Delta(x) \\
-\Delta^{*}(x) & E
\end{array}\right) \psi \equiv \mathcal{M} \psi \\
R^{\prime} \sigma_{3}=\left[\mathcal{M}, R \sigma_{3}\right] \quad \begin{array}{l}
\text { Dik'ii equation } \\
\text { Eilenberger equation }
\end{array}
\end{gathered}
$$

